\title{
The Rayleigh Problem for a Third Grade Electrically Conducting Fluid in a Magnetic Field
}

\author{
Tasawar HAYAT ${ }^{a}$, Herman MAMBILI-MAMBOUNDOU ${ }^{b}$, Ebrahim MOMONIAT $^{b}$ \\ and Fazal M MAHOMED ${ }^{b}$ \\ ${ }^{a}$ Department of Mathematics, Quaid-i-Azam University, Islamabad, Pakistan \\ E-mail: t_pensy@hotmail.com \\ ${ }^{b}$ Centre for Differential Equations, Continuum Mechanics and Applications, School of \\ Computational and Applied Mathematics, University of the Witwatersrand, \\ Johannesburg, Private Bag 3, Wits 2050, South Africa \\ E-mail: Ebrahim.Momoniat@wits.ac.za,Fazal.Mahomed@wits.ac.za
}

\begin{abstract}
The influence of a magnetic field on the flow of an incompressible third grade electrically conducting fluid bounded by a rigid plate is investigated. The flow is induced due by the motion of a plate in its own plane with an arbitrary velocity. The solution of the equations of conservation of mass and momentum balance are obtained analytically. We also present numerical solutions with particular choices of the magnetic field and boundary conditions. Emphasis has been given to the study of the effects of the magnetic field and non-Newtonian fluid parameters.
\end{abstract}

\section{Introduction}

Visco-elastic flows arise in disparate processes in engineering, science and biology - for example, in polymer processing, coating, ink-jet printing, microfluidics, geological flows in the earth's mantle, hemodynamics and the flow of synovial fluid in joints. Modelling viscoelastic flows is important for understanding and predicting the behaviour of processes and thus for designing optimal flow configurations and for selecting operating conditions. The literature on hydrodynamic visco-elastic flows is now quite extensive. However, the literature on the magnetohydrodynamic (MHD) non-Newtonian flows is not large. Reference may be made to the recent interesting studies on the topic by Hayat et al. [1-5].

In all these studies, the applied magnetic field is taken as constant. In this paper we derive an important steady state solution. More recently, Abd-el-Malek et al. [6] obtained the solution of the Rayleigh problem for a power law fluid by taking the magnetic field's strength proportional to $t^{-\frac{1}{2}}$ where $t$ is the time. Wafo Soh [7] extended the analysis of Abd-el-Malek et al. [6] by waving the restriction on $t$. These two papers were two important contributions to the analysis of the effect of time dependent magnetic fields on the 
flow.

Although the power-law model adequately fits the shear stress and shear rate measurement for non-Newtonian fluids, it is not capable of predicting the normal stress effects that lead to phenomena like "die-swell" and "rod-climbing" [8]. Due to this fact, researchers prefer to invoke third grade fluid models. The MHD flow of visco-elastic fluids has applications in different fields. An important field is electromagnetic propulsion. Some fluids with thixotropic behaviour help in the flow of blood, coating of paper, plastic extrusion and lubrication with heavy oils and greases.

Motivated by these analyses, in the present study we extend Wafo Soh's [7] analysis to the flow of a third grade fluid. A further motivation for studying the Rayleigh problem is its close relation with boundary layer growth. Section 2 contains the basic equations. Section 3 deals with the formulation of the problem. Analytical solutions are presented in Sections 4 and 5. Numerical solutions are presented in Section 6. Section 7 contains the concluding remarks.

\section{Basic equations}

The fundamental equations governing the MHD flow of an incompressible electrically conducting fluid are the field equations

$$
\operatorname{div} \mathbf{V}=0, \quad \rho \frac{d \mathbf{V}}{d t}=\operatorname{div} \mathbf{T}+\mathbf{J} \times \mathbf{B}, \quad \mathbf{J}=\sigma(\mathbf{E}+\mathbf{V} \times \mathbf{B})
$$

where $\mathbf{V}$ is the fluid velocity, $\rho$ is the density of the fluid, $\sigma$ is the fluid electrical conductivity, $\mathbf{J}$ is the current density, $\mathbf{B}$ is the magnetic induction so that $\mathbf{B}=\mathbf{B}_{\mathbf{0}}+\mathbf{b}\left(\mathbf{B}_{\mathbf{0}}\right.$ and $\mathbf{b}$ are the applied and induced magnetic fields respectively), $d / d t$ is the material time derivative and $\mathbf{T}$ is the Cauchy stress tensor which for a third grade fluid satisfies the constitutive equation

$$
\begin{aligned}
& \mathbf{T}=-p \mathbf{I}+\mu \mathbf{A}_{1}+\alpha_{1} \mathbf{A}_{2}+\alpha_{2} \mathbf{A}_{1}^{2}+\beta_{1} \mathbf{A}_{3}+\beta_{2}\left[\mathbf{A}_{1} \mathbf{A}_{2}+\mathbf{A}_{2} \mathbf{A}_{1}\right]+\beta_{3}\left(\operatorname{tr} \mathbf{A}_{1}^{2}\right) \mathbf{A}_{1}, \\
& \mathbf{A}_{1}=\operatorname{grad} \mathbf{V}+(\operatorname{grad} \mathbf{V})^{\mathrm{T}}, \\
& \mathbf{A}_{n}=\frac{d \mathbf{A}_{n-1}}{d t}+\mathbf{A}_{n-1} \operatorname{grad} \mathbf{V}+(\operatorname{grad} \mathbf{V})^{\mathrm{T}} \mathbf{A}_{\mathrm{n}-1}, \mathrm{n} \geq 2,
\end{aligned}
$$

where the isotropic stress $p \mathbf{I}$ is due to the constraint of incompressibility, $\mu$ denotes the dynamic viscosity, $\alpha_{i}(i=1,2), \beta_{i}(i=1-3)$ are the material constants, $T$ indicates the matrix transpose and $\mathbf{A}_{i}(i=1-3)$ are the first three Rivlin-Ericksen tensors. Moreover, the Clausius-Duhem inequality and the result that the Helmholtz free energy is minimum at equilibrium provide the following restrictions [9]:

$$
\mu \geq 0, \alpha_{1} \geq 0, \beta_{1}=\beta_{2}=0, \beta_{3} \geq 0,\left|\alpha_{1}+\alpha_{2}\right| \leq \sqrt{24 \mu \beta_{3}} .
$$




\section{Mathematical formulation}

Consider the unidirectional flow of a third grade fluid, obeying equations (2.1) and (2.3), maintained above a non-conducting plate by its motion in its own plane with arbitrary velocity $V(t)$. The fluid is magnetohydrodynamic with small magnetic Reynold's number so that the induced magnetic field is negligible. By taking the velocity field $(u(y, t), 0,0)$, the conservation of mass equation is identically satisfied and in the absence of modified pressure gradient the momentum balance equation (2.1) along with equations (2.2) and (2.3) yields

$$
\frac{\partial u}{\partial t}-\nu \frac{\partial^{2} u}{\partial y^{2}}-\alpha \frac{\partial^{3} u}{\partial t \partial y^{2}}-\epsilon\left(\frac{\partial u}{\partial y}\right)^{2} \frac{\partial^{2} u}{\partial y^{2}}+M H^{2}(t) u=0
$$

where

$$
\nu=\frac{\mu}{\rho}, \alpha=\frac{\alpha_{1}}{\rho}, \epsilon=\frac{6 \beta_{3}}{\rho}, M=\frac{\sigma \bar{\mu}^{2}}{\rho}, \mathbf{H}_{0}=\frac{\mathbf{B}_{0}}{\bar{\mu}} .
$$

In equations (3.1) and (3.2) $\nu$ is the dynamic viscosity and $\bar{\mu}$ is the magnetic permeability of the fluid. By neglecting the modified pressure gradient the dependance of (3.1) on $\alpha_{2}$ has been removed.

The relevant boundary and initial conditions are

$$
\begin{aligned}
& u(0, t)=V(t), \quad t>0, \\
& u(y, t) \rightarrow 0 \text { as } y \rightarrow \infty, \quad t>0, \\
& u(y, 0)=g(y), \quad 0<y<\infty,
\end{aligned}
$$

where $V(t)$ and $g(y)$ are as yet arbitrary functions. These functions are constrained in the next section when we seek exact solutions using the Lie point symmetries method. Also in Section 6, for the numerical solution, we choose specific functions for $V(t)$ and $g(y)$.

\section{Symmetry analysis}

We present a complete Lie point symmetry analysis of the nonlinear partial differential equation (3.1). We find two cases for which equation (3.1) admits a Lie point symmetry algebra. These algebras are used to reduce the initial and boundary value problem (3.1)(3.3) to solvable form.

An operator

$$
\chi=\tau \frac{\partial}{\partial t}+\xi \frac{\partial}{\partial y}+\eta \frac{\partial}{\partial u},
$$

where $\tau, \xi$ and $\eta$ are functions of $t, y$ and $u$, is a Lie point symmetry generator of the partial differential equation (3.1) if [10]

$$
\left.\chi^{[3]}\left\{u_{t}-\nu u_{y y}-\alpha u_{t y y}-\epsilon u_{y}^{2} u_{y y}+M H^{2}(t) u\right\}\right|_{(3.1)}=0
$$


where

$$
\chi^{[3]}=\tau \frac{\partial}{\partial t}+\xi \frac{\partial}{\partial y}+\eta \frac{\partial}{\partial u}+\eta^{t} \frac{\partial}{\partial u_{t}}+\eta^{y} \frac{\partial}{\partial u_{y}}+\eta^{y y} \frac{\partial}{\partial u_{y y}}+\eta^{t y y} \frac{\partial}{\partial u_{t y y}}
$$

is the third prolongation of the operator (4.1) in which the additional coefficient functions satisfy

$$
\begin{aligned}
& \eta^{t}=D_{t}(\eta)-u_{t} D_{t}(\tau)-u_{y} D_{t}(\xi), \\
& \eta^{y}=D_{y}(\eta)-u_{t} D_{y}(\tau)-u_{y} D_{y}(\xi), \\
& \eta^{y y}=D_{y}\left(\eta^{y}\right)-u_{t y} D_{y}(\tau)-u_{y y} D_{y}(\xi), \\
& \eta^{t y y}=D_{t}\left(\eta^{y y}\right)-u_{t y y} D_{t}(\tau)-u_{y y y} D_{t}(\xi)
\end{aligned}
$$

with total derivative operators given by

$$
\begin{aligned}
& D_{y}=\frac{\partial}{\partial y}+u_{y} \frac{\partial}{\partial u}+u_{t y} \frac{\partial}{\partial u_{t}}+\cdots \\
& D_{t}=\frac{\partial}{\partial t}+u_{t} \frac{\partial}{\partial u}+u_{t t} \frac{\partial}{\partial u_{t}}+\cdots .
\end{aligned}
$$

The determining equation (4.2), after separation with respect to the partial derivatives and their powers, gives rise to an overdetermined system of linear homogeneous partial differential equations for the coefficient functions $\tau, \xi$ and $\eta$. Solution of this system gives $\xi=a_{1}$, where $a_{1}$ is a constant, and that $H^{2}$ is constrained by the ordinary differential equation

$$
\frac{d H^{2}}{d t}+\frac{2 \beta a_{3} e^{2 \beta t}}{a_{2}+a_{3} e^{2 \beta t}} H^{2}=\frac{2 \beta^{2} a_{3} e^{2 \beta t}}{M\left(a_{2}+a_{3} e^{2 \beta t}\right)},
$$

where $a_{2}, a_{3}$ are further constants and $\beta=\nu / \alpha$.

The solution for $H^{2}$ gives two sets of Lie point symmetries depending on the values of the constants in the ordinary differential equation (4.4).

\subsection{Case 1: $a_{3}=0, a_{2} \neq 0$}

Equation (4.4) yields $H^{2}=C_{1}$ where $C_{1}$ is a constant and the solutions for the coefficient functions are

$$
\xi=a_{1}, \quad \tau=a_{2}, \quad \eta=a_{4} e^{-M H^{2} t},
$$

where $a_{4}$ is a constant. 
4.2 Case 2: $a_{2}=0, a_{3} \neq 0$

When $a_{2}=0, a_{3} \neq 0, H^{2}=\beta / M+C_{2} e^{-2 \beta t}$ where $C_{2}$ is a constant and the Lie point symmetry coefficients are

$$
\xi=a_{1}, \quad \tau=a_{3} e^{2 \beta t}, \quad \eta=-a_{3} \beta u e^{2 \beta t}+a_{5} L(t), \beta=\nu / \alpha,
$$

where $a_{5}$ is a constant with

$$
L(t)=\exp \left(-M \int_{0}^{t} H^{2}(s) d s\right) .
$$

Thus we obtain two sets of three-dimensional Lie algebras, generated in each case by

Case 1:

$$
X_{1}=\frac{\partial}{\partial y}, \quad X_{2}=\frac{\partial}{\partial t}, \quad X_{3}=L(t) \frac{\partial}{\partial u},
$$

Case 2:

$$
X_{1}=\frac{\partial}{\partial y}, \quad X_{2}=e^{2 \beta t} \frac{\partial}{\partial t}-\beta u e^{2 \beta t} \frac{\partial}{\partial u}, \quad X_{3}=L_{2}^{\prime}(t) \frac{\partial}{\partial u}, \beta=\nu / \alpha
$$

\section{Physical invariant solutions}

Given the generator (4.1) the invariant solution corresponding to $\chi$ is obtained by solving the characteristic system

$$
\frac{d y}{\xi}=\frac{d t}{\tau}=\frac{d u}{\eta}
$$

We use only the operators which give meaningful physical solutions of the initial and boundary value problem (3.1) and (3.3). This means that only $X_{2}$ in each case is considered.

\subsection{Steady state solution corresponding to Case 1}

The form of the invariant solution in this case corresponding to $X_{2}$ is

$$
u(y, t)=F(y)
$$

which is a steady state solution. The substitution of (5.1) into the partial differential equation (3.1) results in the following reduced second-order ordinary differential equation

$$
\left(\nu+\epsilon\left(F^{\prime}(y)\right)^{2}\right) F^{\prime \prime}(y)-C_{1} M F(y)=0 .
$$


The boundary conditions imply

$$
F(0)=u_{0}, \quad F(\infty)=0,
$$

and

$$
V(t)=u_{0}
$$

where $u_{0}$ is a constant.

Note that no steady state solution exists when the magnetic field is zero as equation (5.2) reduces to $F^{\prime \prime}=0$. Applying the boundary condition (5.3) on this equation results in the trivial solution $F=0$.

The double integration of (5.2) leads to

$$
\int^{F} \frac{ \pm \sqrt{\epsilon} d F}{\sqrt{-\nu \pm \sqrt{\nu^{2}+2 \epsilon\left(C_{1} M F^{2}+\lambda_{2}\right)}}}=y+\lambda_{1},
$$

where $\lambda_{1}$ and $\lambda_{2}$ are constants that need to be fixed by (5.3). We observe that we cannot impose the boundary condition $u(y, 0)=g(y)$ for arbitrary $g(y)$. The expression of vorticity is

$$
\begin{aligned}
w_{z} & =-\frac{d F}{d y} \\
& = \pm \sqrt{\frac{-\nu \pm \sqrt{\nu^{2}+2 \epsilon\left(C_{1} M F^{2}+\lambda_{2}\right)}}{\epsilon}} .
\end{aligned}
$$

We focus our attention on the solution (5.5). For a real solution we require the positive sign in the integrand. From (5.3) the boundary condition $F(0)=u_{0}$ implies

$$
\pm \sqrt{\epsilon} \int^{u_{0}} \frac{d F}{\sqrt{\sqrt{\nu^{2}+2 \epsilon C_{1} M F^{2}+2 \epsilon \lambda_{2}}-\nu}}=\lambda_{1} .
$$

Thus equation (5.5) becomes

$$
\pm \sqrt{\epsilon} \int_{u_{0}}^{F} \frac{d F}{\sqrt{\sqrt{\nu^{2}+2 \epsilon C_{1} M F^{2}+2 \epsilon \lambda_{2}}-\nu}}=y .
$$

We now investigate when the boundary condition $F(\infty)=0$ of (5.3) is satisfied for the solution (5.7). Clearly the integral of (5.7) must be divergent as $F \rightarrow 0$ so as to ensure that $y \rightarrow \infty$. It is seen that if $\lambda_{2} \neq 0$, then the integrand behaves like

$$
\left[\sqrt{\nu^{2}+2 \epsilon \lambda_{2}}-\nu\right]^{-1 / 2} \text { as } F \rightarrow 0
$$

which means that the integral in (5.7) is convergent as $F \rightarrow 0$. For $\lambda_{2}=0$, the integral in (5.7) is divergent since the left hand side of (5.7) is

$$
\begin{gathered}
\\
\pm\left(\frac{\epsilon}{\nu}\right)^{1 / 2} \int_{u_{0}}^{F} \frac{d F}{\sqrt{\sqrt{1+2 \epsilon \nu^{-2} C_{1} M F^{2}}-1}} \\
= \pm\left(\frac{\nu}{C_{1} M}\right)^{1 / 2} \int_{u_{0}}^{F}\left(\frac{1}{F}+O(F)\right) d F \text { as } F \rightarrow 0 \\
= \pm\left(\frac{\nu}{C_{1} M}\right)^{1 / 2} \ln \left(\frac{F}{\left.u_{0}\right)}\right)+O\left(F^{2}\right) \text { as } F \rightarrow 0 .
\end{gathered}
$$


This tends to infinity if we take the negative sign. Therefore the solution (5.7) subject to the boundary conditions (5.3) is

$$
\left(\frac{\epsilon}{\nu}\right)^{1 / 2} \int_{F}^{u_{0}} \frac{d F}{\sqrt{\sqrt{1+2 \epsilon \nu^{-2} C_{1} M F^{2}}-1}}=y \text {. }
$$

The vorticity (5.6) upon using (5.8) becomes

$$
w_{z}=\left(\frac{\nu}{\epsilon}\right)^{1 / 2} \sqrt{\sqrt{1+2 \epsilon \nu^{-2} C_{1} M F^{2}}-1} .
$$



Figure 1: Solution of (5.11) and (5.12) for $M^{*}=1$.

We now write the equation (5.2), the solution (5.8) and the vorticity (5.9) in dimensionless form. The scaling transformations

$$
y=u_{0}\left(\frac{\epsilon}{\nu}\right)^{1 / 2} \bar{y}, \quad F=u_{0} \bar{F}
$$

results in equation (5.2) and the boundary conditions (5.3) becoming

$$
\left(1+\left(\frac{d \bar{F}}{d \bar{y}}\right)^{2}\right) \frac{d^{2} \bar{F}}{d \bar{y}^{2}}-M^{*} \bar{F}=0
$$

and

$$
\bar{F}(0)=1, \quad \bar{F}(\infty)=0,
$$

respectively, where

$$
M^{*}=C_{1} M u_{0}^{2} \frac{\epsilon}{\nu^{2}}
$$


is the Hartmann number. So the equation (5.11) depends only on one parameter, viz. the Hartmann number $M^{*}$. Similarly, under the scaling transformation (5.10) the solution (5.8) becomes

$$
\int_{\bar{F}}^{1} \frac{d \bar{F}}{\sqrt{\sqrt{1+2 M^{*} \bar{F}^{2}}-1}}=\bar{y} .
$$

The equation (5.14) is the solution of the boundary value problem (5.11) and (5.12). By means of the scaling

$$
w_{z}=\left(\frac{\nu}{\epsilon}\right)^{1 / 2} \bar{w}_{z}
$$

the vorticity, in terms of the Hartmann number, is

$$
\bar{w}_{z}=\sqrt{\sqrt{1+2 M^{*} \bar{F}^{2}}-1} .
$$

We present the solution of (5.11) and (5.12) for $M^{*}=1$ in Figure 1 and various values of $M^{*}$ in Figure 2. We plot the graph of the vorticity against $\bar{F}$ in Figure 3.

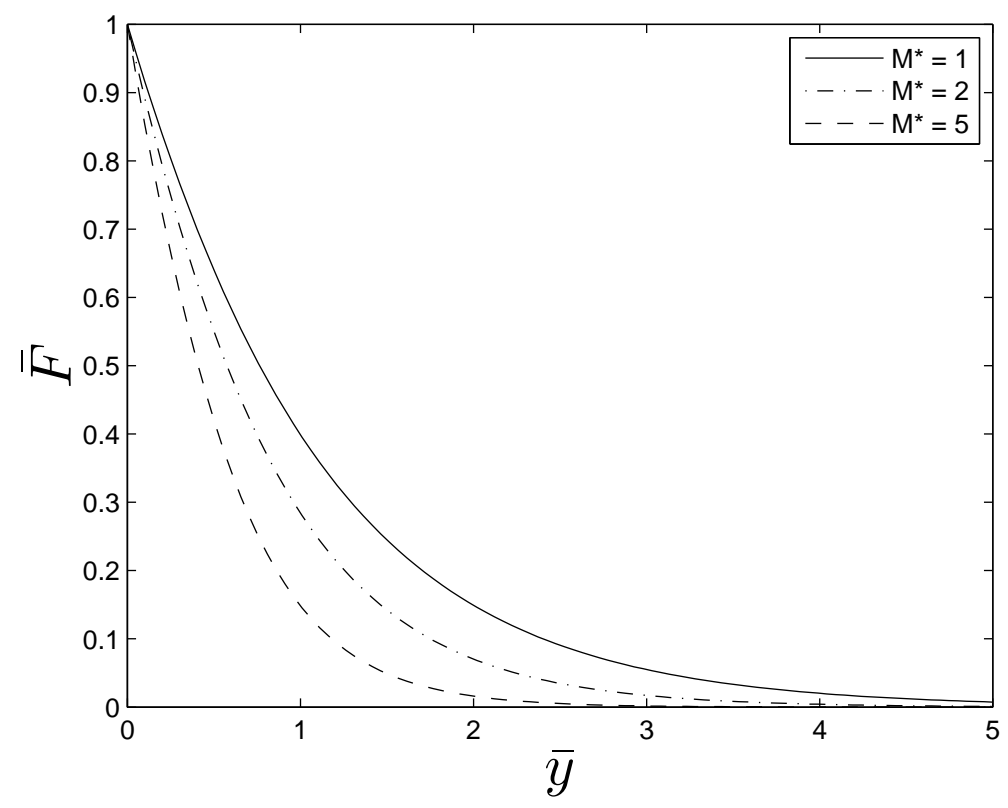

Figure 2: Solution of (5.11) and (5.12) varying $M^{*}$.

We have plotted Figures 1 and 2 to observe the behaviour of the flow when varying different emerging parameter $M^{*}$. Figure 1 presents the solution of the problem consisting of Eqs. (5.11) and (5.12). This indicates that $\bar{F}$ decreases as $\bar{y}$ increases. It can be noted from Figure 2 that the velocity decreases when the Hartmann number $M^{*}$ increases.

We have also plotted the vorticity (5.16) as a function of the velocity in Figure 3 and it is seen that the vorticity increase with the velocity. In Figure 4, we represent the effect of the Hartmann number $M^{*}$ on the vorticity and it shows that the vorticity increases as the Hatmann number increases. 


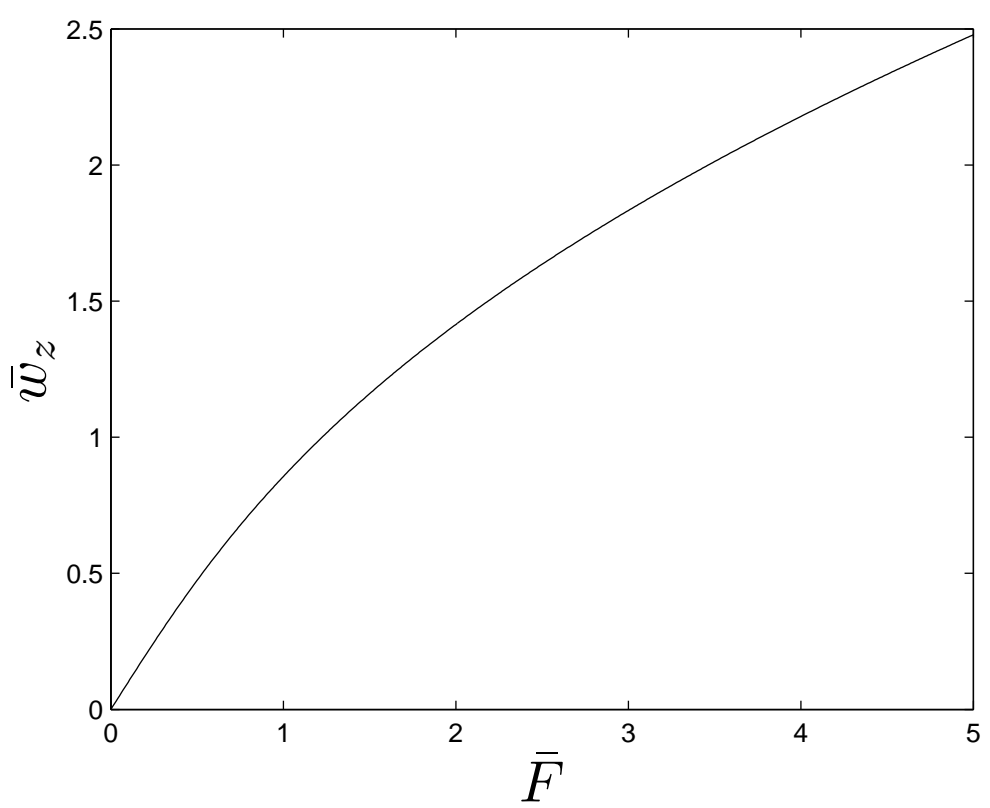

Figure 3: Solution of (5.16) for $M^{*}=1$.

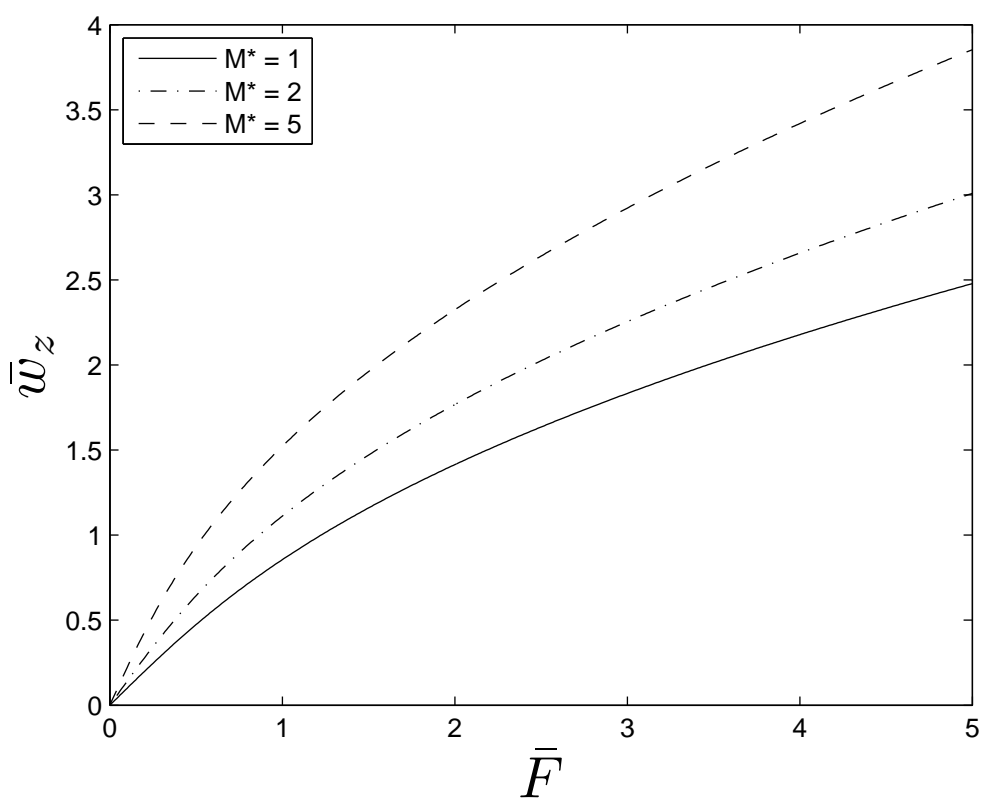

Figure 4: Solution of (5.16) varying $M^{*}$. 


\subsection{Invariant solution corresponding to Case 2}

The invariant solution for this case for $X_{2}$ is given by

$$
u(y, t)=F(y) e^{-\beta t} .
$$

The insertion of (5.17) into (3.1) gives the reduced equation

$$
\gamma\left(F^{\prime}(y)\right)^{2} F^{\prime \prime}(y)-F(y)=0
$$

where $\gamma=\epsilon /\left(C_{2} M\right)$. Through Eqs. (3.3) and (5.17) and selecting $V(t)=C_{2} e^{-\beta t}$, the corresponding boundary conditions are

$$
F(0)=C_{2}, \quad F(\infty)=0 .
$$

To integrate the boundary value problem (5.18) and (5.19), we let

$$
F^{\prime}(y)=K(F)
$$

and substitute (5.20) into (5.18) to obtain

$$
F-\gamma K^{3} \frac{d K}{d F}=0
$$

The integration of (5.21) gives

$$
K(F)=\left(\frac{2}{\gamma} F(y)^{2}+B_{1}\right)^{1 / 4}
$$

where $B_{1}$ is a constant. Equation (5.20) together with (5.22) yields

$$
\frac{d F}{d y}=\left(\frac{2}{\gamma} F(y)^{2}+B_{1}\right)^{1 / 4} .
$$

Thus

$$
\int^{F}\left(\frac{2}{\gamma} F^{2}+B_{1}\right)^{-1 / 4} d F=y+B_{2},
$$

where $B_{2}$ is another constant.

Now we impose the boundary conditions (5.19) on the solution (5.24). The boundary condition $F(0)=C_{2}$ of (5.19) imposed on the solution (5.24) yields

$$
\int^{C_{2}} \frac{d F}{\left(B_{1}+\frac{2}{\gamma} F^{2}\right)^{1 / 4}}=B_{2} .
$$

Therefore with this boundary condition, solution (5.24) becomes

$$
\int_{C_{2}}^{F} \frac{d F}{\left(B_{1}+\frac{2}{\gamma} F^{2}\right)^{1 / 4}}=y
$$




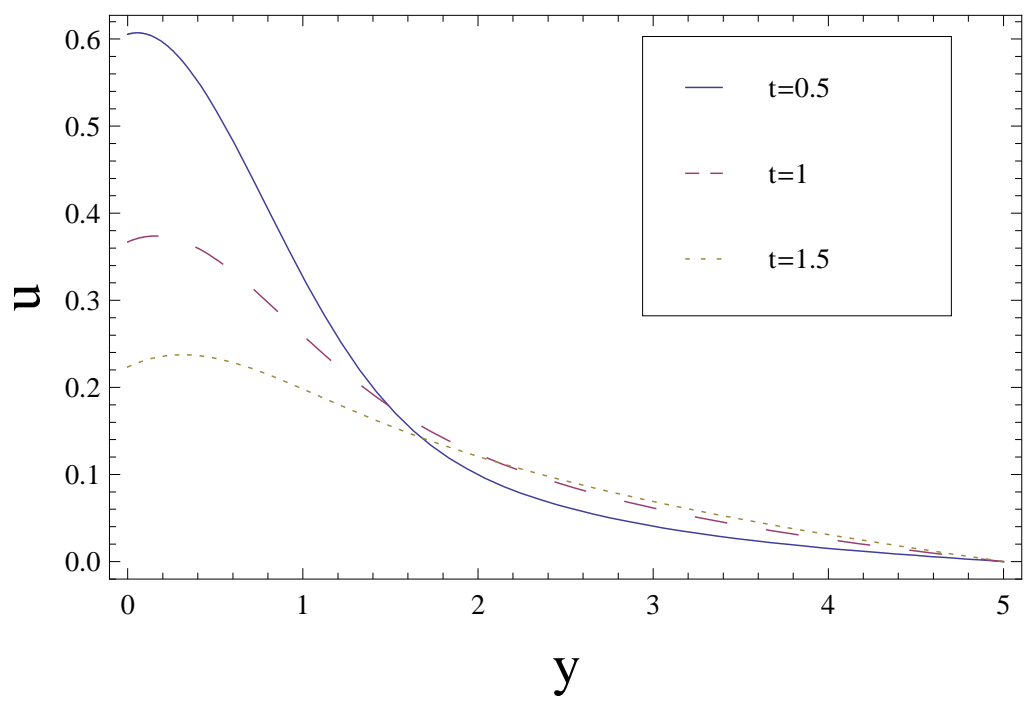

Figure 5: Numerical solution of (3.1) when there is no magnetic field $(H=0)$, with $\epsilon=1.1$, $\nu=1.85, \alpha=1, M=1, V(t)=e^{-t}$ and $g(y)=e^{-y^{2}}$.

Now we invoke $F(\infty)=0$ of (5.19) on the solution (5.25). We first consider the case $B_{1} \neq 0$. The integral (5.25) needs to be divergent as $F \rightarrow 0$ for $y \rightarrow \infty$. For small $F$ the left hand side of (5.25) is convergent. Hence the boundary condition $F(\infty)=0$ is not satisfied. For the case $B_{1}=0$, the solution (5.25) gives

$$
\left(\frac{\gamma}{2}\right)^{1 / 4}\left[2 F^{1 / 2}-C_{2}^{1 / 2}\right]=y
$$

which means that the integral is convergent. Thus the solution (5.25) does not satisfy the boundary condition $F(\infty)=0$.

Therefore for this case we do not obtain a time-dependent solution using symmetry.

\section{Numerical solution of the PDE (3.1)}

In this section we present numerical solutions of the partial differential equation (3.1) subject to (3.3) at various times for different values of the magnetic field and for some emerging parameters.

In Figures 5 to 7 , we have plotted numerically the velocity profile for different values of time, when the magnetic field is first taken to be zero (Figure 5), then constant (Figure 6 ) and dependent on time (Figure 7 ). In the case of $H=0$, the velocity decreases and then increases when the value of $t$ is increased. For each of the curves there is increase of the velocity before decay due to velocity of plate decreasing. The velocities in Figures 5-7 are similar in a qualitative sense. However the shape of velocity differs slightly depending on the form of the magnetic field considered. It can be said that the shape of the velocity is more parabolic in the case when the strength of the magnetic field is non zero. Finally the variation of $\epsilon$ on the velocity is seen in Figure 8. It shows that velocity first decreases slightly and then increases when $\epsilon$ is increased. 


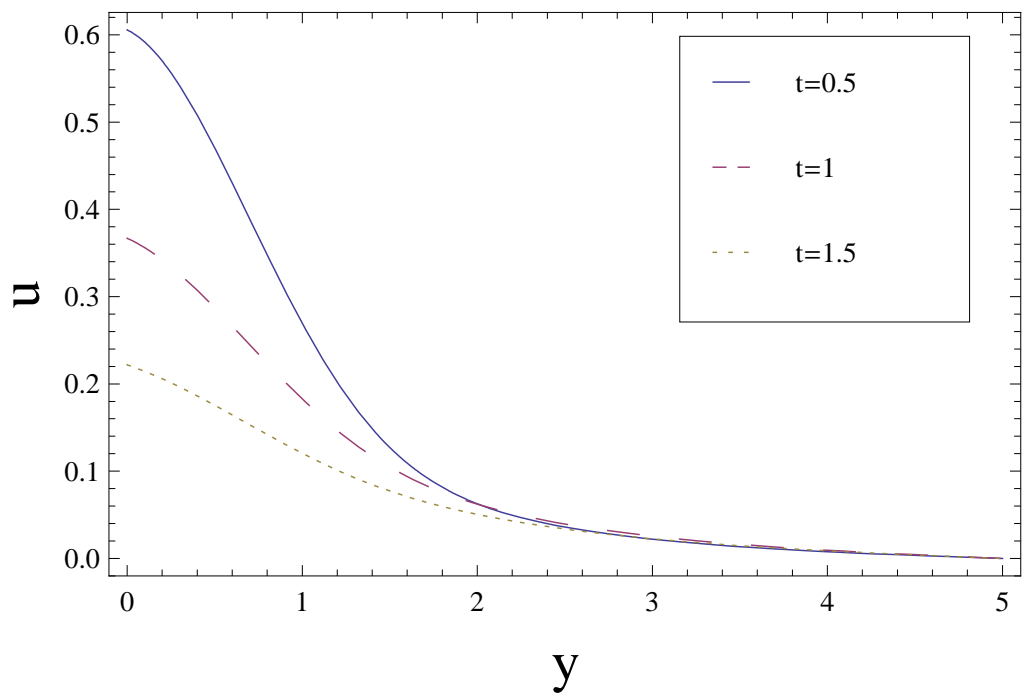

Figure 6: Numerical solution of (3.1) when the magnetic field is taken constant $(H=1)$, with $\epsilon=1.1, \nu=1.85, \alpha=1, M=1, V(t)=e^{-t}$ and $g(y)=e^{-y^{2}}$.

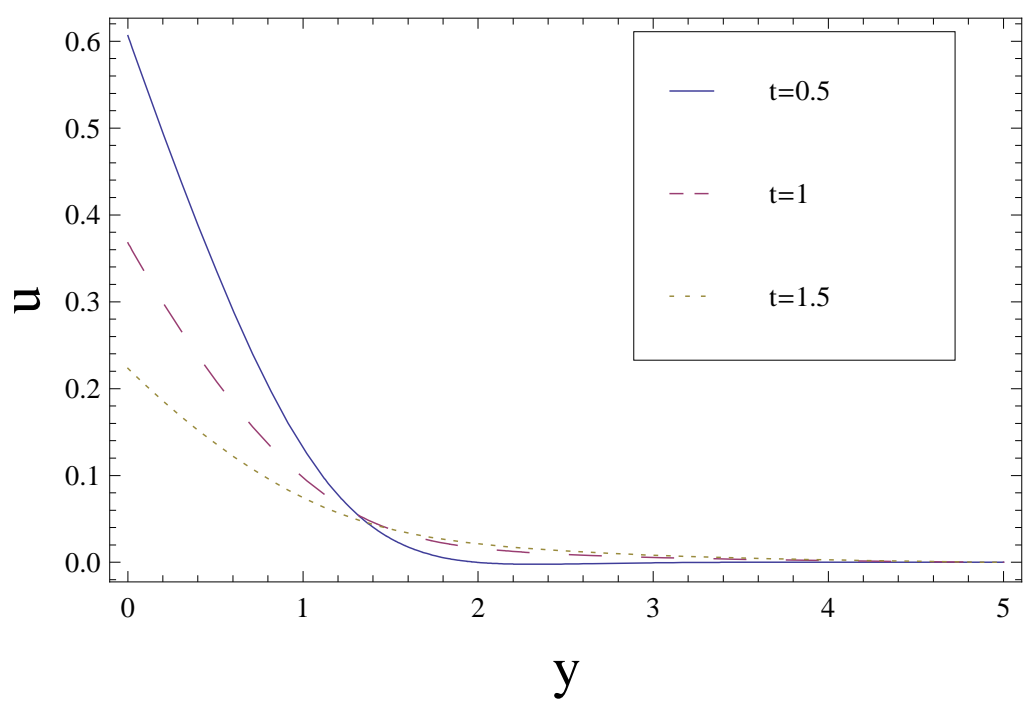

Figure 7: Numerical solution of (3.1) for a time dependent magnetic field $\left(H=1+2 e^{-2 t}\right)$, with $\epsilon=1.1, \nu=1.85, \alpha=1, M=1, V(t)=e^{-t}$ and $g(y)=e^{-y^{2}}$. 


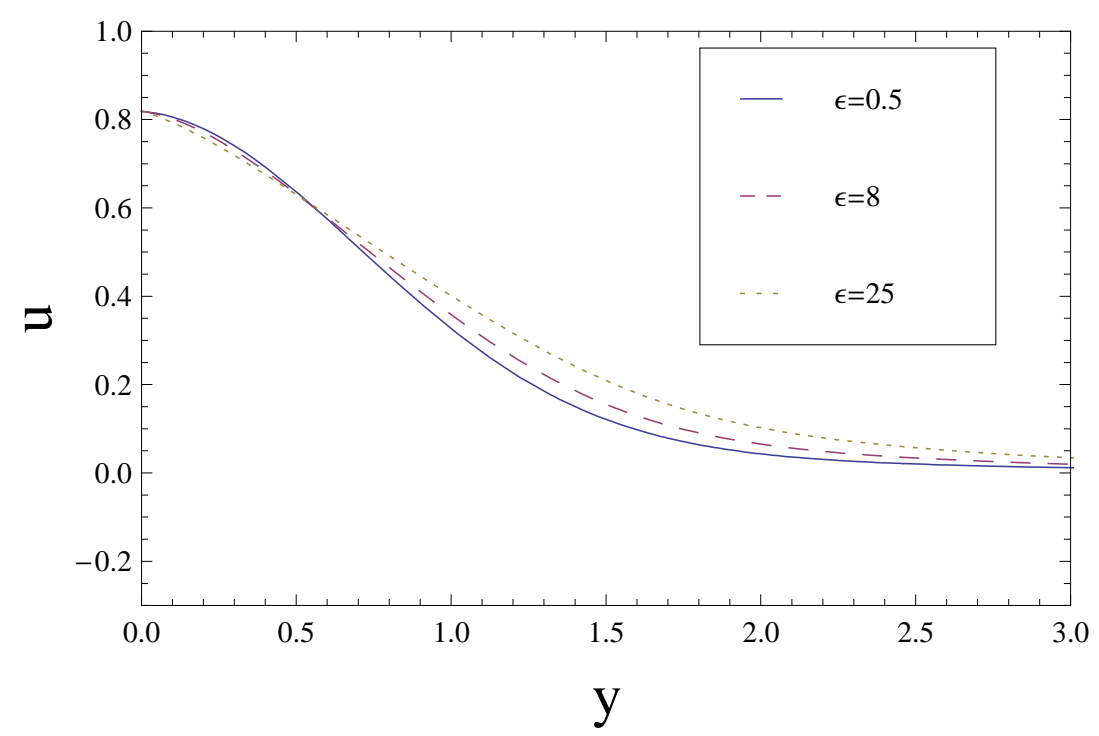

Figure 8: Numerical solution of (3.1) varying $\epsilon$, with $\nu=1.85, H=1+2 e^{-2 t}, \alpha=1, M=1$, $t=0.2, V(t)=e^{-t}$ and $g(y)=e^{-y^{2}}$.

\section{Concluding remarks}

In this paper we have considered the Rayleigh problem of an incompressible electrically conducting non-Newtonian fluid of third grade with magnetic field. After having formulated the problem, we obtained a non-linear third-order partial differential equation. The Lie symmetry approach was employed for the reduction of this equation. We obtained a first-order differential equation that the magnetic field $H$ had to satisfy in order for the existence of Lie point symmetries. Two cases arose and one symmetry was invoked for each case. Invoking the relevant symmetry, we were able to reduce the partial differential equation to a second-order non-linear ordinary differential equation for each of the cases. In the first case, we presented the steady state solutions for various values of the emerging parameter and in the second case, the analytical solution that was obtained did not satisfy the boundary conditions. Thus we have shown that static solutions only exist for $H \neq 0$ by using symmetry methods. Finally, we presented numerical solutions of the partial differential equation with a choice of variable magnetic field as well as suitable boundary conditions in Figures 5 to 8. We briefly comment on the characteristic diffusion distance. The velocity (vorticity) diffuses a characteristic distance $(\nu t)^{1 / 2}$ in time $t$. This is clearly illustrated in Figures 5 to 8 . This may explain why $u$ decays significantly after $t \approx 1$ and not, e.g., $t \approx 10$.

Acknowledgments. FMM acknowledges a Visiting Professorship from the Higher Education Commission of Pakistan and the Department of Mathematics, Quaid-i-Azam University, Islambad, Pakistan during which the final part of this work was completed. EM acknowledges support from the National Research Foundation, South Africa, under grant number 2053745. We also thank one of the referee's for useful and constructive comments which have improved the paper. 


\section{References}

[1] Hayat T, Hutter K, Asghar S and Siddiqui, A. M., MHD flows of an Oldroyd-B fluid, Math. Comp. Modelling. 36, (2002) 987-995.

[2] Hayat T, Nadeem S and Asghar S, Hydromagnetic Couette flow of an Oldroyd-B fluid in a rotating system, Int. J. Eng. Sci. 42, (2004) 65-78 .

[3] Hayat T, Hutter K,Nadeem S and Asghar S, Unsteady hydromagnetic flow of a conducting second grade fluid, Z. Angew. Math. Phys. 55, (2004) 626-641.

[4] Hayat T, Khan M and Asghar S, Magnetohydrodynamic flow of an Oldroyd 6constant fluid, Appl. Math. and Comp. 155, (2004) 417-425 .

[5] Hayat T, Asghar S, Siddiqui A M and Haroon T, Unsteady MHD flow due to non-coaxial rotations of a porous disk and a fluid at infinity, Acta Mech. 151, (2001) $127-134$.

[6] Abdel-el-Malek M B, Badran N A and Hassan H S, Solution of the Rayleigh problem for power law non-Newtonian conducting fluid via group method, Int. J. Eng. Sci. 40, (2002) 1599-1609 .

[7] Wafo Soh C, Invariant solutions of the unidirectional flow of an electrically charged power law non-Newtonian fluid over a flat plate in presence of a transverse magnetic field, Comm. in Non-Linear Sci. and Numerical Simultaion 10, (2005) 537-548 .

[8] Schowalter W R, Mechanics of non-Newtonian fluids, Pergamon, New York (1978).

[9] Fosdick R L and Rajagopal K R, Thermodynamics and stability of fluids of third grade, Proc. Roy. Soc. London. 339, (1980) 351-377 .

[10] Bluman G W and Kumei S, Symmetries and Differential Equations, Springer, New York, (1989) 